\title{
EVALUACIÓN DEL CUMPLIMIENTO DE LAS RECOMENDACIONES DE LA RESOLUCIÓN 0412/00 EN RECIÉN NACIDOS ATENDIDOS EN UN CENTRO DE ATENCIÓN PRIMARIA EN LA CIUDAD DE MANIZALES (COLOMBIA), 2011-2012, ESTUDIO DE CORTE TRANSVERSAL
}

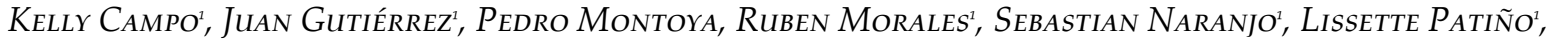 \\ Yair Piedrahitari, Jose Castaño MSc', Oscar Villegas, MD, MSc ${ }^{3}$ \\ ${ }^{1}$ Estudiante de X semestre Programa de Medicina, Universidad de Manizales. \\ ${ }^{2}$ Profesor Titular, Director Centro de Investigaciones, Facultad de Ciencias de la Salud, \\ Universidad de Manizales, Manizales, Caldas, Colombia. \\ ${ }^{3}$ Profesor asociado, Programa de Medicina, Universidad de Manizales, Manizales Colombia. \\ Correspondencia: jcast@umanizales.edu.co
}

Recibido: Febrero 6 de 2014 Aceptado: Mayo 28 de 2014

\begin{abstract}
Resumen
Objetivo: Evaluar la evolución del recién nacido según los cuidados de puericultura citados en la Resolución 0412 del Ministerio de la Protección Social (Colombia) al momento del nacimiento y al tercer día de nacido.

Materiales y métodos: Es un estudio de corte transversal retrospectivo, que se realizó con datos obtenidos de 1202 historias clínicas de atención al nacimiento y control al tercer día en el año 2012 de niños nacidos en el centro ASSBASALUD ESE (Manizales, Colombia). Se evaluaron variables de la madre, variables del parto, variables del neonato al nacimiento y al 3 día.

Resultados: La edad promedio de la madre de 23 años, el 95,1\% asistieron a los controles prenatales. Al momento del nacimiento se encontró que al $98.8 \%$ de los recién nacidos se les colocó brazalete de identificación, también al 98,4\% de los RN se les aplicó las vacunas (BCG, anti-hepatitis B), en el 83,9\% de los partos se realizó un pinzamiento tardío del cordón umbilical; al 99,6\% profilaxis ocular, 99,7\% profilaxis muñón umbilical. Al control del tercer día se observó que el 13,4\% de los recién nacidos presentaron ictericia, el 3,8\% presentaron anormalidades al examen físico siendo la conjuntivitis la más frecuente con un $0,7 \%$ y el $90,1 \%$ asistieron a este control.

Conclusiones: En los hallazgos de esta investigación se concluye que en los cuidados que se le brinda a esta población, se cumplen los parámetros establecidos por la Resolución 0412 del Ministerio de la Protección Social de la República de Colombia.

Palabras clave: Recién nacido, distocia, parto normal, lactancia materna.
\end{abstract}




\title{
NEWBORN ASSESSMENT AND MONITORING THE THIRD DAY OF LIFE IN A PRIMARY CARE CENTER IN THE MANIZALES CITY (COLOMBIA). 2011-2012, CROSS-SECTIONAL STUDY
}

\begin{abstract}
Objective: Evaluate the evolution of newborn care as childcare resolution 0412 cited in Ministry of Social Protection at birth and on the third day of life.

Materials and methods: This is a retrospective cross-sectional study, which was performed with 1202 data from medical records of birth care and control on the third day in 2012 children born in the center ASSBASALUD ESE (Manizales, Colombia). The study evaluated 37 variables were classified as maternal variables, birth variables, and NB variables to 3 days.

Results: The average age of the mother of 23 years, $95.1 \%$ attended prenatal care. At birth it was found that $100 \%$ of infants were placed identification bracelet, also at $100 \%$ of newborns were administered vaccines (vsg, hepatitis B), $83.9 \%$ of births performed delayed cord clamping. The control of the third day was observed that $13.4 \%$ of the newborns had jaundice, $3.8 \%$ of the newborns had abnormalities on physical examination remain the most frequent conjunctivitis with $0.7 \%$ and $90,1 \%$ attended this control.

Conclusions: In the findings of this research concludes that ASSBASALUD ESE institution meets the parameters of 0412 resolution of health and social protection ministry of the Colombian republic.
\end{abstract}

Keywords: Newborn, dystocia, natural childbirth, breastfeeding.

\section{AVALIAÇÃO DA APLICAÇÃO DAS RECOMENDAÇÕES DA RESOLUÇÃO 0412/00 EM RECÉM NASCIDOS EM UM CENTRO DE ATENÇÃO PRIMARIA NA CIDADE DE MANIZALES (COLÔMBIA) 2011-2012, ESTUDO DE CORTE TRANSVERSAL}

\begin{abstract}
Resumo
Objetivo: Avaliar a evolução do recém nascido segundo as recomendações de puericultura da resolução 0412 do Ministério da Proteção Social (Colômbia) no momento do nascimento $e$ ao terceiro dia de nascido.

Materiais e Métodos: Estudo de coorte transversal-retrospectivo o qual foi feito em $2012 \mathrm{com}$ dados obtidos de 1202 historias clinicas de atenção ao nascimento e controle ao terceiro dia, de crianças nascidos no centro ASSBASALUD ESE (Manizales, Colômbia). Foram avaliadas variáveis da mãe, de parto, do neonato ao nascimento e ao terceiro dia.

Resultados: A idade meia das mães foi de 23 anos; o 95\% tiveram controles pré-natais. Ao momento do nascimento, o $98.8 \%$ dos recém nascidos tinham pulseira de identificação, o $98.4 \%$ tinham vacinas (BCG, anti-hepatitis B); o $83.9 \%$ tinham pinçamento tardio do cordão umbilical;
\end{abstract}


o $99.6 \%$ profilaxia ocular e o $99.7 \%$ profilaxia do cordão umbilical. No controle do terceiro dia (90,1\% das crianças) se observou que o $13,4 \%$ dos recém nascidos apresentaram icterícia. Além disso, o $3.8 \%$ apresentaram anormalidades ao exame físico, sendo a conjuntivites a mais frequente $(0,7 \%)$.

Conclusões: Os dados deste estúdio concluem que os cuidados que são oferecidos a esta população cumprem os parâmetros estabelecidos pela resolução 0412 do Ministério da Proteção Social da Republica da Colômbia.

Palavras-chave: Recém nascido, distócia, parto normal, lactancia materna

\section{Introducción}

La mortalidad en menores de un año en Colombia (2002 - 2004) se estimó en 26,4 por mil nacidos vivos ${ }^{1}$, cifra elevada, que afecta negativamente a la salud pública ${ }^{1}$.De este grupo etario, los recién nacidos (RN) aportan cerca del 60\% de los decesos ${ }^{1,2}$. Al obedecer muchos de ellos a causas susceptibles de identificar e intervenir, esta proporción puede disminuirse aplicando, las acciones de Promoción y Prevención recomendadas en la Resolución 0412 de $2000^{1,2}$.

Se dice que de una gestación normal, se deben derivar una madre y un bebé sanos ${ }^{2}$, mediante el control de los factores de riesgo para garantizar una adecuada adaptación al medio externo confirmada por la valoración completa e inmediata después del parto, durante la hospitalización $y$ en el control a realizar al RN en las primeras 72 ho$\mathrm{ras}^{3}$. Partiendo del conocimiento de la norma, se pretende documentar por medio de este estudio, no sólo evaluar la ejecución de la misma sino sus beneficios, en medio de las múltiples variables que afectan su aplicación, que permitan acumular criterios que sean recomendables en la atención materno infantil al obtener buenos niveles de evidencia científica.

Son fundamentales las acciones médicas y de puericultura desde el mismo momento de ocurrido la expulsión fetal con maniobras como el contacto piel a piel de manera precoz, estimulación dérmica, valoración del APGAR buscando una excelente transición de la vida intra a la extrauterina.

Otras prácticas incluyen las profilaxis ocular, umbilical y de la enfermedad hemorrágica del RN, pinzamiento del cordón como lo recomiendan las circunstancias, ${ }^{4-6}$ evitar la hipotermia, identificar al RN ${ }^{7-10}$ y muchas más ${ }^{10,11}$ y previo al alta, garantizar la asignación de una cita de control al tercer día, para valorar los cambios fisiológicos y detectar las eventuales alteraciones no reconocidas durante la evolución intrahospitalaria.
En un estudio realizado en Soledad y Barranquilla (Atlántico, Colombia) en $2007^{12}$ se observó una alta proporción de $\mathrm{RN}$ en los que no se cumple la norma, principalmente por omisión en los profesionales que participan en la atención de los RN. También se puede tomar como un reto para mejorar la atención que redunde en un buen pronóstico para los RN, tal como lo menciona el Fondo de Población de las Naciones Unidas en su plan de maternidad y nacimiento seguros. Si se tiene en cuenta que hay pocos trabajos de corte similar que describen la realidad de esta condición y, que para Manizales (Caldas) no existen, se propuso adelantar esta investigación en una comunidad pobre y vulnerable, en época vigente, que demanda cuanto antes un diagnóstico y, resultados oportunos y efectivos.

La succión y la alimentación del RN a base de lactancia materna exclusiva (LME) se constituye en el pilar fundamental para incentivar y vigilar. Si no hay contraindicación, la forma preferible es la alimentación al pecho. ${ }^{1,2,7,8,12-15}$ ya que la deserción sigue siendo alta. ${ }^{14-17}$.El control a las 72 horas es importante porque en el se valora la adecuada evolución de RN. El médico y la familia del bebé, para lograr una mayor participación y éxito, deben capacitarse en los cuidados de puericultura. ${ }^{18,19} \mathrm{Al}$ respecto, Rodríguez-Cué$l l a r^{20}$ et al en un estudio realizado en el hospital Dr. José Eleuterio González en el que revisaron 488 RN atendidos en el hospital universitario en México, encontraron que 338 no acudieron al seguimiento. Los motivos identificados para incumplir la cita fueron la falta a su vez de control prenatal, el tabaquismo materno y, el bajo nivel académico entre otros; el hecho de que las mujeres estuvieran casadas disminuyó el riesgo de inasistencia al control del RN.

En la India la Organización Mundial de la Salud ${ }^{21}$ (OMS) realizó un estudio en 2008 para evaluar las prácticas domésticas que pueden afectar la salud neonatal, desde la perspectiva de los cuidadores y trabajadores de la salud, buscar las "señales de peligro" y determinar la proporción de los cuidadores que reconocen los distintos componen- 
tes de atención en el RN. Se encontró que el llanto continuo es reportado como una manifestación común de la enfermedad neonatal y fue motivo para buscar ayuda para el $23 \%$ de las personas encuestadas.

Por las consideraciones anteriores se planteó la presente investigación que pretende describir las características de los bebés nacidos en una Institución prestadora de salud (IPS) del estado colombiano, de primer nivel de complejidad, que atiende población afiliada al sistema subsidiado en una ciudad intermedia (Manizales, Colombia), y observar la evolución del RN, bajo el cuidado de sus padres, siguiendo las recomendaciones y los cuidados de puericultura de acuerdo a lo establecido en las normas ${ }^{1}$ desde el momento del nacimiento hasta los 3 días de vida.

\section{Materiales y métodos}

Se realizó un estudio de corte transversal analítico. La población de estudio consistió en 1202 niños que nacieron en ASSBASALUD ESE (Empresa Social del Estado, que presta servicios de salud de baja complejidad en Manizales, Colombia) en la sede Clínica San Cayetano. La población son todos los bebés nacidos en esta institución en el período 2011-2012. La información se obtuvo, exclusivamente, de las historias clínicas de los niños y sus madres. Criterios de inclusión: niño nacido en ASSBASALUD ESE sede San Cayetano. Criterios de exclusión fueron: recién nacidos que son remitidos a un hospital de tercer nivel por patologías de base, $\mathrm{RN}$ pretérmino, $\mathrm{RN}$ con malformaciones congénitas, $\mathrm{RN}$ con bajo peso y talla al nacer.

En la realización del estudio se tuvieron en cuenta las siguientes variables: estado civil materno (Soltero (no estable), casado y unión libre (estable)), control prenatal (número de consultas), procedencia (urbano, rural), edad de la madre, estrato social (1-6), nivel educativo (ninguno, primaria, secundaria, universidad), grupo sanguíneo $(A, B, O, A B), R h$ (positivo, negativo), aseguramiento al sistema de salud (no asegurada, subsidiado, contributivo, otro), género del recién nacido (femenino, masculino), peso al nacer (gr), edad gestacional del recién nacido (pretérmino, término, postérmino), contacto piel a piel al nacimiento (recibe, no recibe), PAI al nacimiento (BCG, antihepatitis B), puntaje APGAR (primer minuto, cinco minutos), tiempo de pinzamiento de cordón umbilical (inmediato, precoz, tardío), presencia de ictericia al tercer día (si, no), profilaxis ocular al nacer (si, no), profilaxis con vitamina $\mathrm{K}$ al nacer (si, no), desarrollo neurológico (succión, llanto, diuresis, excreción de meconio), examen físico inicial (peso, talla, perímetro cefálico, perímetro torácico), fecha de nacimiento.
La revisión de las historias clínicas se llevó a cabo entre septiembre del 2012 y febrero del 2013.

Referente a los análisis estadísticos las variables medidas en escala nominal se describieron mediante tablas de frecuencia; las variables medidas en escala numérica mediante promedio y desviación estándar. Se calcularon límites de confianza para los valores de las variables medidas tanto en escala nominal como numérica. Las bases de datos se elaboraron en el programa Excel 2010 (Microsoft Corp.) y fueron analizadas empleando los programas estadísticos SPSS 21 (IBM Corp.) y Epiinfo 3.5.5 (Centers for disease control and prevention, (CDC)).

En la presente investigación se cumplieron todas las normas éticas de ley vigentes en Colombia. El proyecto fue enviado a ASSBASALUD ESE y aprobado por el Comité de Ética e Investigación de esa institución con comentarios.

\section{Resultados}

En la Tabla 1 se describe la población de gestantes incluidas en el presente trabajo. En total 1201 historias clínicas válidas: el 63,2\% corresponden a nacimientos en 2011 y $36,8 \%$ en 2012 . El promedio de edad de las gestantes fue de 23 años, el 63,5\% de las participantes gozaban de unión estable (lc 95\%: 60,6\%-66,2\%), el 98,4\% procedían de Manizales (lc 95\%: 97,5\%-99\%). Se identifica una población de recursos limitados, el 78,2\% vive en el estrato social 2 (lc 95\%: 75,4\%-80,8\%) y consecuentemente, el 93,2\% pertenecen al régimen subsidiado (lc 95\%: 91,6\%-94,5\%), nivel educativo mediano, $74,1 \%$ han cursado hasta secundaria (lc 95\%: 71,5\%-76,6\%).

Asistieron a los controles prenatales el 95,1\% (lc 95\%: 93,7\%-96,2\%), de las cuales 15,2\% (lc 95\%: 13,2\%-17,4\%) acudieron en 7 oportunidades que se constituyó en la concentración más común. La hemoclasificación materna más registrada fue $\mathrm{O}^{+}$, con un 58,8\% (lc 95\%: 55,9\%-61,7\%); al 94,3\% de las gestantes se les realizó el VDRL durante el embarazo (lc 95\%: 92,8\%-95,6\%).

En la tabla 2, se describen las variables relacionadas con el nacimiento: se observa que el $46,1 \%$ de las participantes recibieron acompañamiento durante el parto (lc 95\%: 43,2\%-49\%), el cual fue eutócico en el 98,7\% de los casos (lc 95\%: 97,9\%-99,3\%). El 86,7\% de RN presentaron un APGAR normal al primer minuto de nacido (lc 95\%: $84,6 \%-88,55)$, el puntaje 8 fue el de mayor frecuencia, con 47,8\% (lc 95\%: 45\%-59,7\%); a los 5 minutos se amplió el APGAR normal al 99,2\% de los RN (lc 95\%: 98,5599,6\%), con un 10 como índice de mayor regularidad, en el 93,1\% (lc 95\%: 91,4\%-94,4\%). 
Tabla 1. Variables referentes a las gestantes participantes en el estudio sobre valoración del recién nacido, y seguimiento hasta el $3^{\circ}$ día en clínicas de ASSBASALUD ESE, 2012.

\begin{tabular}{|c|c|c|c|}
\hline Variables & Niveles & $\mathbf{N}$ & $\%$ \\
\hline \multirow{2}{*}{ Año de nacimiento } & 2012 & 757 & 63,2 \\
\hline & 2011 & 440 & 36,9 \\
\hline \multirow{3}{*}{ Estado civil } & Estable & 749 & 63,5 \\
\hline & No estable & 431 & 36,5 \\
\hline & Faltantes & 21 & \\
\hline \multirow{5}{*}{ Procedencia } & Manizales & 1191 & 99,1 \\
\hline & Neira & 3 & 2 \\
\hline & Manizales área rural & 5 & 0,5 \\
\hline & Pácora & 1 &, 1 \\
\hline & Quinchia-Risaralda & 1 &, 1 \\
\hline \multirow{7}{*}{$\begin{array}{l}\text { Edad de la madre } \\
\text { (años) }\end{array}$} & Válidos & 1201 & \\
\hline & Promedio & 23,1 & \\
\hline & LC95\% LI & 22,7 & \\
\hline & LC95\% LS & 23,4 & \\
\hline & Des. Est. & 5,9 & \\
\hline & Mínimo & 14 & \\
\hline & Máximo & 48 & \\
\hline \multirow{5}{*}{ Estrato Social } & 2 & 736 & 78,2 \\
\hline & 3 & 127 & 13,5 \\
\hline & 1 & 69 & 7,3 \\
\hline & 4 & 9 & 1 \\
\hline & Faltantes & 260 & \\
\hline \multirow{5}{*}{ Seguridad Social } & Subsidiado & 1110 & 93,2 \\
\hline & Otro & 74 & 6,2 \\
\hline & Contributivo & 4 & ,3 \\
\hline & No asegurado & 3 &, 3 \\
\hline & Faltantes & 10 & \\
\hline \multirow{6}{*}{ Nivel educativo } & Bachillerato & 856 & 74,1 \\
\hline & Primaria & 179 & 15,5 \\
\hline & Ninguno & 68 & 5,9 \\
\hline & Educación superior & 40 & 4,5 \\
\hline & Otro & 12 & 1 \\
\hline & Faltantes & 46 & \\
\hline \multirow{3}{*}{$\begin{array}{l}\text { Asistencia control } \\
\text { prenatal }\end{array}$} & $\mathrm{Si}$ & 1130 & 95,1 \\
\hline & No & 58 & 4,9 \\
\hline & Faltantes & 14 & \\
\hline \multirow{18}{*}{$\begin{array}{l}\text { Número de contro- } \\
\text { les prenatales }\end{array}$} & 7 & 170 & 15,2 \\
\hline & 8 & 155 & 13,8 \\
\hline & 6 & 152 & 13,5 \\
\hline & 4 & 130 & 11,6 \\
\hline & 5 & 128 & 11,4 \\
\hline & 3 & 102 & 9,1 \\
\hline & 9 & 96 & 8,6 \\
\hline & 1 & 92 & 8,2 \\
\hline & 2 & 87 & 7,8 \\
\hline & 11 & 8 & 0,7 \\
\hline & Faltantes & 391 & \\
\hline & Válidos & 1120 & \\
\hline & Promedio & 5,4 & \\
\hline & LC95\% LI & 5,4 & \\
\hline & Lc95\% LS & 6 & \\
\hline & Des. Est. & 2,4 & \\
\hline & Mínimo & 1 & \\
\hline & Máximo & 11 & \\
\hline \multirow{9}{*}{ Hemoclasificación } & $\mathrm{O}^{+}$ & 684 & 58,8 \\
\hline & $\mathrm{A}^{+}$ & 322 & 27,7 \\
\hline & O- & 60 & 5,2 \\
\hline & $\mathrm{B}+$ & 59 & 5,1 \\
\hline & A- & 24 & 2,1 \\
\hline & $\mathrm{AB}^{+}$ & 9 & 0,8 \\
\hline & B- & 4 & 0,3 \\
\hline & AB- & 1 & 0,1 \\
\hline & Faltantes & 39 & \\
\hline \multirow{3}{*}{ VDRL } & $\mathrm{Si}$ & 1116 & 94,3 \\
\hline & No & 67 & 5,7 \\
\hline & Faltantes & 19 & \\
\hline
\end{tabular}

Tabla 2. Variables referentes al momento del nacimiento en población de un centro de atención primaria (ASSBASALUD ESE) en la ciudad de Manizales entre el 2011 y 2012

\begin{tabular}{|c|c|c|c|}
\hline Variables & Nivel & $\mathbf{N}$ & $\%$ \\
\hline \multirow{3}{*}{$\begin{array}{l}\text { Acompañante } \\
\text { durante el parto }\end{array}$} & No & 643 & 53,9 \\
\hline & SI & 550 & 46,1 \\
\hline & Faltantes & 9 & \\
\hline \multirow{3}{*}{ Tipo de parto } & Eutócico & 1181 & 98,7 \\
\hline & Distócico & 15 & 1,3 \\
\hline & Faltantes & 6 & \\
\hline \multirow{3}{*}{$\begin{array}{l}\text { Género del recién } \\
\text { nacido }\end{array}$} & Masculino & 628 & 52,6 \\
\hline & Femenino & 567 & 47,4 \\
\hline & Faltantes & 7 & \\
\hline \multirow{5}{*}{$\begin{array}{l}\text { Edad gestacional } \\
\text { (semanas) }\end{array}$} & Válidos & 1190 & \\
\hline & Promedio & 38,9 & \\
\hline & Límite inferior & 38,8 & \\
\hline & Límite superior & 39 & \\
\hline & Desviación estándar & 1,68 & \\
\hline \multirow{9}{*}{ Rh Recién Nacido } & $\mathrm{O}+$ & 703 & 59,9 \\
\hline & $\mathrm{A}^{+}$ & 308 & 26,3 \\
\hline & $\mathrm{B}^{+}$ & 77 & 6,5 \\
\hline & $\mathrm{O}-$ & 46 & 3,9 \\
\hline & A- & 24 & 2,1 \\
\hline & $\mathrm{AB}^{+}$ & 11 & 0,9 \\
\hline & B- & 6 & 0,5 \\
\hline & AB- & 0 & 0 \\
\hline & Faltantes & 27 & \\
\hline \multirow{3}{*}{ Huellas } & $\mathrm{Si}$ & 1193 & 99,5 \\
\hline & No & 6 & 0,5 \\
\hline & Faltantes & 3 & \\
\hline \multirow{3}{*}{ Baño } & No & 826 & 68,9 \\
\hline & $\mathrm{Si}$ & 373 & 31,1 \\
\hline & Faltantes & 3 & \\
\hline \multirow{3}{*}{ Contacto piel a piel } & $\mathrm{Si}$ & 1189 & 99,2 \\
\hline & No & 8 & 0,8 \\
\hline & Faltantes & 4 & \\
\hline \multirow{3}{*}{ Brazalete } & $\mathrm{Si}$ & 1188 & 98,8 \\
\hline & No & 8 & 0,7 \\
\hline & Faltantes & 6 & \\
\hline \multirow{4}{*}{ PAI } & BCG-Hepatitis B & 1176 & 98,4 \\
\hline & Hepatitis B & 15 & 1,3 \\
\hline & BCG & 4 & 0,3 \\
\hline & Faltantes & 6 & \\
\hline \multirow{12}{*}{ APGAR 1 minuto } & Apgar normal & 1033 & 86,7 \\
\hline & Apgar bajo & 159 & 13,3 \\
\hline & Faltantes & 10 & \\
\hline & 8 & 571 & 47,8 \\
\hline & 9 & 430 & 36,1 \\
\hline & 7 & 117 & 9,8 \\
\hline & 10 & 32 & 2,7 \\
\hline & 6 & 30 & 2,5 \\
\hline & 4 & 4 & ,3 \\
\hline & 5 & 4 & 3 \\
\hline & 2 & 2 &, 2 \\
\hline & 3 & 2 & ,2 \\
\hline \multirow{9}{*}{ APGAR 5 minutos } & Apgar normal & 1186 & 99,2 \\
\hline & Apgar bajo & 9 &, 8 \\
\hline & Faltantes & 7 & \\
\hline & 10 & 1113 & 93,1 \\
\hline & 9 & 60 & 5 \\
\hline & 8 & 11 & ,9 \\
\hline & 7 & 4 &, 3 \\
\hline & 6 & 3 & ,3 \\
\hline & 5 & 2 & ,2 \\
\hline
\end{tabular}

(Continúa en la página siguiente..) 
Tabla 2. Variables referentes al momento del nacimiento en población de un centro de atención primaria (ASSBASALUD ESE) en la ciudad de Manizales entre el 2011 y 2012. (Continuación)

\begin{tabular}{|c|c|c|c|}
\hline Variables & Nivel & $\mathbf{N}$ & $\%$ \\
\hline \multirow{4}{*}{$\begin{array}{l}\text { Pinzamiento del } \\
\text { cordón umbilical }\end{array}$} & Tardío & 1003 & 83,9 \\
\hline & Inmediato & 174 & 14,6 \\
\hline & Precoz & 18 & 1,5 \\
\hline & Faltantes & 7 & \\
\hline \multirow{3}{*}{$\begin{array}{l}\text { Acompañamiento } \\
\text { conjunto }\end{array}$} & $\mathrm{Si}$ & 1195 & 99,6 \\
\hline & No & 5 & 0,4 \\
\hline & Faltantes & 2 & \\
\hline \multirow{3}{*}{ TSH } & $\mathrm{Si}$ & 1193 & 99,5 \\
\hline & No & 6 & 0,5 \\
\hline & Faltantes & 3 & \\
\hline \multirow{3}{*}{ Profilaxis ocular } & $\mathrm{Si}$ & 1193 & 99,6 \\
\hline & No & 5 & 0,4 \\
\hline & Faltantes & 4 & \\
\hline \multirow{3}{*}{$\begin{array}{l}\text { Profilaxis muñón } \\
\text { umbilical }\end{array}$} & $\mathrm{Si}$ & 1196 & 99,7 \\
\hline & No & 4 & 0,3 \\
\hline & Faltantes & 2 & \\
\hline \multirow{2}{*}{$\begin{array}{l}\text { Producto para } \\
\text { profilaxis de muñón } \\
\text { umbilical }\end{array}$} & Alcohol & 1200 & 100 \\
\hline & Faltantes & 2 & \\
\hline \multirow{3}{*}{$\begin{array}{l}\text { Profilaxis } \\
\text { vitamina K }\end{array}$} & $\mathrm{Si}$ & 1190 & 99,3 \\
\hline & No & 8 & 0,7 \\
\hline & Faltantes & 4 & \\
\hline \multirow{2}{*}{ Succión } & $\mathrm{Si}$ & 1190 & 100 \\
\hline & Faltantes & 11 & \\
\hline \multirow{2}{*}{ Llanto } & $\mathrm{Si}$ & 1193 & 100 \\
\hline & Faltantes & 8 & \\
\hline \multirow{2}{*}{ Diuresis } & $\mathrm{Si}$ & 1186 & 100 \\
\hline & Faltantes & 15 & \\
\hline \multirow{2}{*}{ Meconio } & $\mathrm{Si}$ & 1159 & 100 \\
\hline & Faltantes & 42 & \\
\hline \multirow{4}{*}{$\begin{array}{l}\text { Nivel de Peso al } \\
\text { nacer }\end{array}$} & Normal & 1148 & 95,8 \\
\hline & Bajo & 30 & 2,5 \\
\hline & Alto & 20 & 1,7 \\
\hline & Faltantes & 3 & , 2 \\
\hline \multirow{5}{*}{ Peso al nacer (grs) } & Válidos & 1198 & \\
\hline & Promedio & 3194 & \\
\hline & LC95\% LI & 3172 & \\
\hline & Lc95\% LS & 3217 & \\
\hline & Des. Est. & 395 & \\
\hline \multirow{4}{*}{ Nivel de Talla } & Normal & 1039 & 86,5 \\
\hline & Baja & 156 & 13 \\
\hline & Alta & 3 &, 2 \\
\hline & Faltantes & 3 & , 2 \\
\hline \multirow{5}{*}{ Talla al nacer $(\mathrm{cm})$} & Válidos & 1198 & \\
\hline & Promedio & 48,7 & \\
\hline & LC95\% LI & 48,5 & \\
\hline & Lc95\% LS & 48,8 & \\
\hline & Des. Est. & 2,4 & \\
\hline \multirow{4}{*}{$\begin{array}{l}\text { Nivel Perímetro } \\
\text { cefálico }\end{array}$} & Normal & 1071 & 89,2 \\
\hline & Bajo & 94 & 7,8 \\
\hline & Alto & 30 & 2,5 \\
\hline & Faltantes & 6 &, 5 \\
\hline & Válidos & 1195 & \\
\hline & Promedio & 33,5 & \\
\hline Perímetro cefálico & LC95\% LI & 33,4 & \\
\hline & Lc95\% LS & 33,6 & \\
\hline & Des. Est. & 1,6 & \\
\hline & Validos & 1180 & \\
\hline & Promedio & 33,2 & \\
\hline Permetro toracico & LC95\% LI & 32,7 & \\
\hline & LC95\% LS & 33,7 & \\
\hline & Des. Est. & 8,6 & \\
\hline
\end{tabular}

El 52,6\% de los nacimientos correspondió a varones (lc 95\%: 49,7\%-55,4\%), el promedio de edad gestacional fue de 38,93 semanas (lc 95\%: 24,5\%-29,6\%); el 59,9\% de ellos presentaban tipo de sangre O+ (lc 95\%: 57,1\%62,7\%). El 99,5\% se identifican con la toma de huellas plantares (lc 95\%: 98,9\%-99,8\%) y con brazaletes el 98,8\% (lc 95\%: 98,6\%-99,7\%).

Los cuidados inmediatos al nacimiento incluyeron el baño o limpieza con pañitos tibios al 31,1\% de los bebés (lc 95\%: 28,5\%-33,8\%), contacto piel a piel entre madre e hijo en el 99,2\% de ellos (lc 95\%: 98,5\%-99,6\%), pinzamiento tardío del cordón umbilical en el 83,9\% (lc 95\%: 81,7\%-85,9\%) e inmunización al 98,4\% de los bebés con BCG y con antihepatitis B (lc 95\%: 97,5\%-99). Se ejecutaron 3 profilaxis en la sala de partos a saber: a. Ocular, que se hizo al 99,6\% de los bebés (lc 95\%: 99\%-99,8\%), b. Del muñón umbilical al 99,7\% (lc 95\%: 99,1\%-99,9\%) y c. De la enfermedad hemorrágica del RN con vitamina K al 99,3\% (lc 95\%: 98,6\%99,7\%). Al 99,5\% se les tomó muestra de sangre del cordón para realizarle la prueba de TSH para tamizaje de patología tiroidea (lc 95\%: 98,9\%-99,8\%).

Del mismo modo, la valoración antropométrica incluye: 1 . Pesaje, el 95,8\% (lc 95\%: 94,5\%-96,9\%) de los RN presentaron peso en límites normales, con promedio de peso al nacer de $3194 \mathrm{gr}$, 2. Talla; el 86,5\% (lc 95\%: 84.6\%-88,6\%) presentaron talla en límites normales, con $48,7 \mathrm{~cm}$ el promedio, 3. El perímetro cefálico fue normal en $89,2 \%$ de los bebés (lc 95\%: 87,7\%-91,3\%), con promedio de 33,5 cm y 4. Perímetro torácico con un promedio de $33,2 \mathrm{~cm}$.

Como imprescindible para el alta de neonato se valoran la presencia de succión y llanto enérgicos, confirmar la diuresis y que se compruebe haber excretado meconio, acciones que se documentaron en el 100\% de los recién nacidos,

En la Tabla 3, se despliegan las variables del RN referentes al control del tercer día: el 90,7\% asistieron a este control (lc 95\%: 88,4\%-91,5\%) en el cual se comprueba que tanto el peso, la talla y el perímetro cefálico encontrados en esta cita, y al relacionarlos con las cifras presentadas al nacer, se encuentran en límites normales -95,9\% (lc 95\%: 91\%: - 94,7\%), 86,3\% (lc 95\%: 87,5\%:-91,3\%), 77,7\% (lc 95\%: $74,8 \%-80,3 \%)$ respectivamente. Igualmente los promedios de peso ( $3177 \mathrm{gr})$, de talla $(49 \mathrm{~cm})$, de perímetro cefálico $(34,5 \mathrm{~cm})$ y de perímetro torácico $(33,7 \mathrm{~cm})$, hallados al tercer día de nacimiento, son normales y concordantes con los encontrados al nacer.

El 96,3\% (lc 95\%: 95\%-97,3\%) de los RN al tercer día reciben lactancia materna exclusiva, $94,7 \%$ se encontraron 
Tabla 3. Variables del recién nacido referentes al control del tercer día en población de un centro de atención primaria en la ciudad de Manizales entre el 2011 y 2012.

\begin{tabular}{|c|c|c|c|}
\hline Variables & Niveles & $\mathbf{N}$ & $\%$ \\
\hline \multirow{2}{*}{$\begin{array}{l}\text { Asistencia a } \\
\text { controles }\end{array}$} & Sí & 1089 & 90,7 \\
\hline & No & 112 & 9,3 \\
\hline \multirow{4}{*}{$\begin{array}{l}\text { Nivel de Peso al } \\
\text { tercer día }\end{array}$} & Normal & 1144 & 95,9 \\
\hline & Bajo & 47 & 3,9 \\
\hline & Alto & 2 &, 2 \\
\hline & Faltantes & 9 & \\
\hline \multirow{5}{*}{ Peso al 3 día (gr) } & Válidos & 1042 & \\
\hline & Promedio & 3177 & \\
\hline & LC95\% LI & 3153 & \\
\hline & Lc95\% LS & 3202 & \\
\hline & Des. Est. & 407 & \\
\hline \multirow{4}{*}{ Nivel de Talla } & Normal & 1030 & 86,3 \\
\hline & Baja & 153 & 12,8 \\
\hline & Alta & 10 & ,8 \\
\hline & Faltantes & 9 & \\
\hline \multirow{5}{*}{ Talla al 3 día (cm) } & Válidos & 1064 & \\
\hline & Promedio & 49 & \\
\hline & LC95\% LI & 48,8 & \\
\hline & Lc95\% LS & 49,2 & \\
\hline & Des. Est. & 2,96 & \\
\hline \multirow{4}{*}{$\begin{array}{l}\text { Nivel de Perímetro } \\
\text { cefálico al } 3 \text { día }\end{array}$} & Normal & 713 & 77,7 \\
\hline & Alto & 196 & 21,4 \\
\hline & Bajo & 196 & 1 \\
\hline & Faltantes & 283 & \\
\hline \multirow{5}{*}{$\begin{array}{l}\text { Perímetro cefálico al } \\
3 \text { día }(\mathrm{cm})\end{array}$} & Válidos & 918 & \\
\hline & Promedio & 34,45 & \\
\hline & LC95\% LI & 34,35 & \\
\hline & Lc95\% LS & 34,56 & \\
\hline & Des. Est. & 1,66 & \\
\hline \multirow{5}{*}{$\begin{array}{l}\text { Perímetro torácico al } \\
3 \text { día }(\mathrm{cm})\end{array}$} & Válidos & 672 & \\
\hline & Promedio & 33,68 & \\
\hline & LC95\% LI & 33,54 & \\
\hline & LC95\% LS & 33,81 & \\
\hline & Des. Est. & 1,76 & \\
\hline \multirow{3}{*}{ Lactancia materna } & Recibe & 1049 & 96,3 \\
\hline & No Recibe & 40 & 3,7 \\
\hline & Faltantes & 112 & \\
\hline \multirow{3}{*}{ Estado de alerta } & Vigoroso & 1081 & 94,7 \\
\hline & Hipoactivo & 60 & 5,3 \\
\hline & Faltantes & 61 & \\
\hline \multirow{3}{*}{ Presencia de ictericia } & No & 985 & 86,6 \\
\hline & $\mathrm{Si}$ & 153 & 13,4 \\
\hline & Faltantes & 64 & \\
\hline \multirow{3}{*}{$\begin{array}{l}\text { Anormalidades al } \\
\text { examen físico }\end{array}$} & No & 1100 & 96,2 \\
\hline & $\mathrm{Si}$ & 44 & 3,8 \\
\hline & Faltantes & 58 & \\
\hline \multirow{3}{*}{$\begin{array}{l}\text { Alteraciones al } \\
\text { examen físico }\end{array}$} & Conjuntivitis & 8 & ,7 \\
\hline & Onfalitis & 3 &, 2 \\
\hline & Apéndices preauriculares & 2 &, 2 \\
\hline
\end{tabular}

vigorosos $(93,2 \%-95,9 \%)$ al momento del examen y el 13,4\% presentaron estigmas de ictericia (lc 95\%: 11,5\%$15,6 \%)$; únicamente se detectaron cifras anormales en el examen físico al 3,8\% (lc 95\%:2,8\%-5,2\%), correspondiendo a la conjuntivitis el hallazgo más prevalente $(0,7 \%)$.

\section{Discusión}

Según la Resolución 0412/00 ${ }^{1}$ del Ministerio de Salud y Protección Social de la República de Colombia, la atención del RN está fundamentada en un conjunto de actividades, cuidados, intervenciones y procedimientos, dirigidos a los bebés durante el proceso de parto y el tiempo inmediatamente después de nacer, reduciendo eventuales riesgos de desequilibrios, daños, complicaciones y procurando el bienestar general del individuo, evitando las múltiples secuelas que causan discapacidad en la niñez y las altas tasas de morbimortalidad perinatal e infantil ${ }^{1}$.

La mayoría de las complicaciones del período neonatal son prevenibles, controlables y tratables ${ }^{21}$, además están asociadas con la salud de la mujer, la calidad de la atención en la gestación, el proceso del parto y el puerperio mediato.

Los partos distócicos de cualquier causa, suelen ser un factor negativo a tener en cuenta. En este estudio el 1,3\% de los trabajos de parto analizados tuvieron un desenlace distócico, 0,8\% con un bajo puntaje de APGAR. En comparación en un estudio realizado por Laffita ${ }^{22}$ et al en el año 2004 en el Hospital América Arias de Cuba se encontró que el $71,26 \%$ de las madres con RN con APGAR bajo presentó un parto distócico, relación que no fue probada en el presente estudio.

El corte del cordón umbilical ha tenido gran relevancia en los últimos años por haber demostrado beneficios para el neonato, se ha clasificado según el tiempo en que se realiza 3 categorías: precoz, habitual y tardío. El pinzamiento tardío se puede decir que es causa de transfusión de niveles altos de ferritina, hemoglobina y hematocrito, llevando así a una disminución en la incidencia de anemia por deficiencia de hierro en el $\mathrm{RN}^{23}$. La presente investigación mostró que en el $83,9 \%$ de los trabajos de parto, se realizó pinzamiento tardío del cordón umbilical. Comparado este resultado con el obtenido en un estudio realizado en Uruguay por Sinavszki ${ }^{24}$ et al, se concluye que esta práctica está correctamente implementada en la comunidad.

En 1974, cuando no se había implementado el PAI, solo el $5 \%$ de los niños aproximadamente habían sido vacunados. Actualmente el $79 \%$ de la población mundial recibe estas vacunas. En el presente estudio el 98,4\% de los RN recibió la vacunación contra BCG y la anti-hepatitis B.

La conjuntivitis neonatal es una enfermedad frecuente, aplicar yodopovidona en cada ojo inmediatamente después del nacimiento es una técnica aprobada para prevenirla ${ }^{25}$. En el presente estudio al 99,7\% de los RN se les realizó adecuada profilaxis ocular. Otro tipo de profilaxis 
importante a realizar en el RN son la del muñón umbilical y la aplicación de vitamina K. Estas se realizaron en el presente estudio en $99,3 \%$ y $99,7 \%$ respectivamente.

En la profilaxis del cordón umbilical este estudio mostró que al 99,7\% de los RN se les aplicó alcohol, que demuestra su eficacia ya que al evaluar los resultados solo el $0,2 \%$ de los RN desarrollaron onfalitis, coincidiendo con el hallado por Duque ${ }^{26}$ et al en el año 1999, que encontraron que el alcohol produce menor reacción inflamatoria y alérgica en el muñón umbilical comparado con las sustancias iodadas.

A través del tamizaje neonatal se identifican varias alteraciones congénitos, metabólicas y endocrinas, que tratadas en forma oportuna disminuyen la morbimortalidad infantil. En el actual estudio se realizó prueba de TSH al 99,5\% de la población, evidenciándose que no hubo casos de hipotiroidismo. Mazzi y Bohrt ${ }^{27}$ en el año 2010, efectuaron un estudio en una población de 2142 neonatos, en el cual al $100 \%$ de la población se le realizó TSH neonatal, encontrándose un caso de hipotiroidismo neonatal, esto indica que el cribado neonatal debe ser adoptado en forma universal ${ }^{27}$.

Al control al 3 día asistieron en el 90,7\% de los casos, en el estudio de Rodríguez-Cuéllar ${ }^{20}$ efectuado en el 2004 se encontró una asistencia de 30,7\%.

En la presente investigación al tercer día el 94,2\% de los RN recibieron LME, estadísticas de la India (UNICEF) estiman que solo el $46 \%$ de los neonatos se alimenta de esta manera. ${ }^{28}{ }^{26} \mathrm{El} 13,4 \%$ de los RN presentaron ictericia al tercer día, en otras poblaciones, por ejemplo en el estudio de Rodríguez ${ }^{29}$ et al en Madrid, en el año 2008 la encontraron en un $60 \%$. A la madre debe explicársele cuando es conveniente que acuda para evaluar la ictericia y se considere tratamiento preventivo.

El contacto piel con piel, entre madre y RN, precoz y prolongado después del parto, se implementó en el 99,2\% de los casos; se ha comprobado que este contacto tiene efectos benéficos para el $\mathrm{RN}^{30}$.

La toma de las medidas antropométricas es una forma efectiva y económica para evaluar el tamaño del cuerpo humano. Según los patrones de crecimiento dados por la $\mathrm{OMS}^{31}$ el peso adecuado para el recién nacido está comprendido entre 2500 y 4000 gramos. En el presente estudio el 95,8\% de los RN evaluados tuvieron peso en límites normales ${ }^{32}$, con promedio al nacer de 3194 gramos. En cuanto a la talla el $86,5 \%$ de los RN la presentaron entre límites normales ${ }^{30}$, con promedio de $49 \mathrm{~cm}$. Referente al perímetro cefálico el $89,6 \%$ de los RN estuvieron entre límites normales ${ }^{30}$ con promedio de $33,5 \mathrm{~cm}$.

Rosales-Amarís ${ }^{12}$ et al efectuaron un estudio publicado en el 2007 en el que trataban de establecer el cumplimiento de la norma de que trata la Resolución 0412/ 2000 en algunos hospitales de Soledad y Barranquilla, encontraron que el APGAR a los 5 minutos fue tomado en el 64,3\% de los casos, la identificación oportuna se realizó en el $50 \%$ de los casos, toma de medidas antropométrica en el $100 \%$, examen físico en un $58 \%$. Resultados en general mejores se presentaron en la presente investigación aunque en esta no se evaluaron los aspectos de atención humanizada, de trabajo en equipo, y capacidad resolutiva que se analizaron en el estudio de Rosales-Amarís. A su vez en la presente investigación se analizaron aspectos no considerados en el estudio de Rosales-Amarís.

Como conclusión del presente estudio se puede afirmar que en general en la institución considerada, en los aspectos evaluados se encontró un buen cumplimiento de lo recomendado por la norma 0412 , que se tuvieron en cuenta en la presente investigación.

Se evidenció incongruencia, más allá de la esperada, en las medidas antropométricas tomadas al momento del nacimiento y al tercer día; posiblemente asociadas a errores por parte del personal de salud o uso inadecuado de los equipos de toma.

En el proceso de realización del estudio y al interpretar los resultados se debe considerar algunas limitantes como la falta de datos en la historia clínica al momento del registro, inadecuada digitación de las medidas antropométricas por parte del personal de salud, uso de instrumentos de tecnología caduca para la recolección de medidas antropométricas la cual hace que los datos obtenidos al nacimiento y 3 días después en el control, sean ambivalentes. Esto se vio reflejado en la incongruencia mencionada entre las medidas antropométricas al nacimiento y al 3 día.

Conflictos de interés: Los autores declaran no tener ningún conflicto de interés en relación al tema del estudio.

Financiación: Universidad de Manizales.

\section{Referencias}

1. Ministerio de Salud y Protección Social de la República de Colombia. Resolución 0412 de 2000. Bogotá D.C.: Ministerio de Salud y Protección Social de la República de Colombia; 2000. 
2. Fariñas A. El control de la oftalmia neonatal gonocócica. Rev Cubana Med Gen Integr 1998;14(5):510-4.

3. Frenk J, Ruelas E, Tapia R, Castañón R, De León ME, González C, et al. Embarazo saludable, parto y puerperio seguros. Recién nacido sano. México DF Secretaría de salud, dirección general de salud reproductiva; 2001

4. Moore ER, Anderson GC, Bergman N. Contacto temprano piel a piel entre las madres y sus recién nacidos sanos (Revisión). Barcelona: John Wile y \& Sons, Ltd; 2007.

5. Blouin B, Penny M, Casapia M, Aguilar E, Silva H, Serene $\mathrm{J}$, et al. Effect of a two-component intervention to change hospital practice from early to delayed umbilical cord clamping in the peruvian amazon. Rev Panam Salud Pública 2011; 29(5):322-328.

6. Doménech E, González N, Rodríguez-Alarcón J. Cuidados generales del recién nacido o sano. Madrid: Asociación Española de Pediatría; 2008.

7. Quiroga A, Chattas G, Castañeda A, Ramirez M, Montes-Bueno $\mathrm{T}$, Iglesias $\mathrm{A}$, et al, Guía práctica clínica de termorregulación en el recién nacido. Buenos Aires: Sociedad Iberoamericana de Neonatologia; 2010.

8. Noguera-Ortiz NY, Rodriguez-Rodríguez MR. Aprendiendo a cuidar al recién nacido: un cuidado congruente con la cultura. Avances en Enfermería 2008; 26 (1):103-111.

9. Martinez M. Recién Nacido Sano y Patológico. Valoración y Cuidados De Enfermería. Lactancia Natural y Artificial. Estimulación Precoz De La Lactancia Natural. En: Actualización en técnicas, procedimientos, cuidados y normativa para enfermería en el ámbito hospitalario y de atención primaria. Alicante: concejo de enfermería de la comunidad valenciana; 2004.

10. Ronald S. Cohen, Ronald J, Stevenson D. Understanding neonatal jaundice: a perspective on causation. Pediatr Neonatol 2010;51(3):143-148.

11. Puckett RM, Offringa M. Prophylactic vitamin K for vitamin $\mathrm{K}$ deficiency bleeding in neonates. Cochrane Database of Systematic Reviews 2000; 4: Art. No.: CD002776. DOI: 10.1002/14651858.CD002776.

12. Rosales-Amarís M, Alvarez-Manduca K, Delgado-Rivero L, Hemer-Narváez MA, Madero-Reales J, Silva-Henao D. Cumplimiento de la norma técnica de atención del recién nacido en hospitales del nivel I y III de Barranquilla y Soledad. Salud Uninorte 2007; 23(1):52-63.

13. Kramer M, Kakuma R. The optimal duration of exclusive breastfeeding a systematic review. Montreal: Departments of Pediatrics and of Epidemiology and Biostatistics, McGill University Faculty of Medicine; 2012.

14. Zanardo V, Svegliado G, Cavallin F, Giustardi A, Cosmi E, Litta $\mathrm{P}$ et al. Elective cesarean delivery: does it have a negative effect on breastfeeding? Birth. 2010; 37:275-9.

15. Barriuso L, Sánchez-Valverde F. Prevalencia de la lactancia materna en el norte de España. ANALES Sis San Navarra 1998, 21 (Supl. 3):13-19.

16. Costas M, Santos S, Godoy C, Martell M. Patrones de succión en el recién nacido de término y pretérmino. Rev Chil Pediatr 2006; 77(2):198-212.

17. Cardona K, Castaño JJ, Hurtado CI, Moreno Mi, Restrepo A, Villegas OA. Frecuencia de la lactancia materna exclusiva, factores asociados y relación con el desarrollo antropométrico y patologías en una población de lactantes atendidos en ASSBASALUD ESE. Manizales (Colombia) 2010-2011. Arch Med (Manizales) 2013; 13 (1):73-87.

18. Darmstadt G, Mohamed H, Balsara Z, Winch P. Impact of clean Delivery -kit use on Newborn umbilical cord and Maternal Puerperal Infections in Egypt. J Health Popul Nutr 2009; 27(6):746-754.

19. Barnett S, Azad K, Barua S, Abrar M. Maternal and Newborn-care Practices during Pregnancy, Childbirth, and the postnatal Period: A comparison in Three rural Districts in Bangladesh. J Health Popul Nutr 2006, 24(4): 394-402.

20. Rodríguez-Cuéllar EA, Marroquín-Escamilla AR, AbregoMoya V, Rodríguez-Balderrama I, Treviño-Garza C. Recién nacidos con egreso temprano. ¿Por qué no acuden al seguimiento? Med Univer 2004; 6(24):176-181.

21. Awasthi S, Verma T, Agarwal M. Signos de peligro de las enfermedades neonatales: impresiones de los cuidadores y de los trabajadores sanitarios en el norte de la India. Bulletin of the World Health Organization 2006; 84(10).

22. Laffita-Batista A, Ariosa JM, Cutié-Sánchez JR. Apgar bajo al nacer y eventos del periparto. Rev Cubana Obstet Ginecol 2004; .30(1):0-0.

23. Raju TN. Timing of umbilical cord clamping after birth for optimizing placental transfusion. Curr Opin Pediatr 2013; 25(2):180-7.

24. Sinavszki M, Sosa N, Silvera F, Díaz-Rossello JL. Clampeo tardío del cordón umbilical: saturación de oxígeno en recién nacidos.Arch Pediatr Urug 2011; 82(3): 141-146.

25 Darling E, Mc Donald H. A Meta-analysis of the Efficacy of ocular Prophylactic agents used for the prevention of gonococcal and Chlamydial OphthalmiaNeonatorum. J Midwifery Womens Health 2010; 5:319-326.

26. Duque M, Lozano D, Ortiz C, Quiñones A. Efecto de la profilaxis umbilical en el recién nacido. Investigación en enfermería. Imagen y Desarrollo 1999. 1(1):55-59.

27. Mazzi-Gonzales de Prada E, Bohrt Terceros V. Cribado neonatal. Rev Bol Ped 2010; 49(3):145-149.

28. WalkerA. Las madres de la india, el país del té, aprenden sobre la importancia de la lactancia materna. Geneva; UNICEF; 2010.http://www.unicef.org/spanish/infobycountry/ india_54058.html:

29. Rodríguez J, Figueras A. Ictericia neonatal. En: Protocolos de neonatología. $2^{\circ}$ ed. Madrid: Asociación Española de Pediatría, Sociedad Española de Neonatología; 2008.

30. Melchor-MarcosJC.Contacto piel con piel en la sala de partos y muerte súbita del recién nacido. Una llamada de atención. Prog Obstet Ginecol 2011; 54:53-4.

31. Fondo de las Naciones Unidas para la Infancia (UNICEF). Evaluación del crecimiento de niños y niñas. Buenos Aires: Fondo de las Naciones Unidas para la Infancia (UNICEF); 2012.

32. De Onís M, Garza C, Victora CG, Onyango AW, Frongillo EA, Martines J. El Estudio Multi-centro de la OMS de las Referencias del Crecimiento: Planificación, diseño y metodología. Food and Nutrition Bulletin 2001; 25(1): S15-S26. 